\title{
Letter
}

\section{Robust Numerical Resolution of Nakamura Crystallization Kinetics}

\author{
Arthur Levy \\ Laboratoire de Thermique et Energie de Nantes, Université de Nantes, Nantes, France \\ Email address: \\ arthur.levy@univ-nantes.fr

\section{To cite this article:} \\ Arthur Levy. Robust Numerical Resolution of Nakamura Crystallization Kinetics. International Journal of Theoretical and Applied \\ Mathematics. Vol. 3, No. 4, 2017, pp. 143-147. doi: 10.11648/j.ijtam.20170304.13
}

Received: December 1, 2016; Accepted: December 16, 2016; Published: October 23, 2017

\begin{abstract}
The numerical prediction of crystallization transformation is of great interest in several applications. One such application is the polymer-forming process. In this short communication, the integration of the widely used Nakamura kinetics is discussed. A robust time integration method is proposed. In order to overcome its singularities, the Nakamura function is thresholded. A convergence analysis provides guidelines for the threshold values and time discretization.
\end{abstract}

Keywords: Crystallization Kinetics, Nakamura, Time Integration, Robust, Phase Change

\section{Introduction}

In recent decades, the numerical simulation of manufacturing processes of polymers and polymer matrix composites has been significantly improved. The latest simulation softwares $[1,2,3]$ can account for several complex coupled non-linear physical phenomena [4]. With the development of these predictive tools, the industry could better understand, control, and eventually optimize the forming processes. today, optimizing the processing windows, in terms of process parameters or material properties, requires quantitative simulation tools. Thus an accurate modeling of fine physical phenomena is needed.

Industrial thermoplastic polymers are often semi-crystalline. They include several widely distributed polymers (such as PE, PA, PET and PP [5, 6]), as well as high technology polymers (aromatic PEEK or PEKK for instance [7]). For these materials, crystallization, which occurs during the forming process, and especially during the solidification phase, plays a critical role in the final quality of the part. For instance, in the case of injection moulding, crystallization determines the liquid/solid transition and thus rules the ejection criteria. In addition, crystallization induces shrinkage and residual stresses.

Therefore, polymer-forming simulation softwares must accurately predict crystallization effects, especially during the cooling phase. An accurate quantitative prediction is a prerequisite for predicting the solidification (or gel) time.

The crystallization of polymer macromolecules depends on both temperature and flow history. A macroscopic homogenized approach consists of defining a continuous crystallinity variable $x$, which evolves from 0 for an amorphous material to a maximum crystallinity $x_{\text {max }}$, specific to the semi-crystalline polymer considered. The degree of crystallization $\alpha$ is also defined as the degree of advancement of the transformation and thus varies from 0 (when $x=0$ ) to 1 (when $x=x_{\text {max }}$ ). The evolution of $\alpha$ is ruled by the crystallization kinetics law [8].

This paper focuses on quiescent thermal crystallization. In fact, temperature appears to be the prevailing driver of crystallization in several forming processes, in which flow-induced crystallization can be neglected.

\section{Method}

\subsection{Problem Definition}

\subsubsection{Avrami Integral Form}

The most common thermal crystallization models are derived from the Avrami model [9]. Under Avrami assumptions (uniform germ location, instantaneous growth after activation, given crystal growth morphology [10]), isothermal crystallization evolution is written: 


$$
\alpha=1-\exp \left(-K_{a v} t^{n}\right)
$$

where $n$ is the Avrami index and $K_{a v}$ the Avrami crystallization kinetics function.

In the seventies, Nakamura et al. [11] extended the Avrami model to account for non-isothermal crystallization. In its initial form, the degree of crystallization was given as the integral form:

$$
\alpha=1-\exp \left[-\left(\int_{0}^{t} K(T) d t\right)^{n}\right]
$$

where $K(T)$ is the Nakamura kinetics crystallization function which is related to the Avrami isothermal kinetics $1 / n$

function $k(T)$ by $K(T)=K_{a v}(T)$. This expression was also obtained by Billon et al. [12, 8].

The Nakamura model proved efficient for modelling the crystallization of different polymer systems [13, 14, 15].

\subsubsection{Differential Form}

Patel \& Spruiell [5] suggested using the differential form of equation (1) which is written

$$
\frac{d \alpha}{d t}=K(T) \times \underbrace{n(1-\alpha)[-\ln (1-\alpha)]^{1-\frac{1}{n}}}_{G(\alpha)}
$$

The Nakamura function $G$ is plotted over $[0,1]$ in Figure 1.

This differential form, a first-order non-linear ordinary differential equation, is more natural to implement and solve numerically, especially in a multiphysical framework $[16,17$, $18,19,20,21,22,23]$. The initial state of the material (amorphous or semi-crystalline) should be given as an initial condition for $\alpha$.

Even though several polymers exhibit more complex crystallization behaviour, the Nakamura law (2) very often appears in the kinetics. This is the case, for instance, with kinetics accounting for secondary crystallization, in which two coupled Nakamura laws can be used [24, 25].

\subsubsection{Artificial Germination}

In its differential form, because $G(0)=0$, an initial fully amorphous state $\alpha(t=0)=0$ would never induce crystallization. Thus, it is common to consider an artificial initial germination

$$
\alpha(t=0)=\varepsilon
$$

where $\varepsilon$ is a small value. To the author's knowledge, its influence has never been fully investigated in the literature and will be discussed below.

\subsection{Time Integration}

Equation 2 is highly non-linear. In the case in which the temperature $T(t)$ (and thus $K(T(t))$ ) depends on time, there is no analytical solution to this equation. Therefore, a numerical integration is required.

The time and degree of crystallization are discretized. In this short communication, a standard first-order incremental integration scheme is considered. At an instant $t_{n}$, the degree of crystallization $\alpha_{n}$ is computed from the previous value $\alpha_{n-1}$. Two extreme and very general integration schemes are considered:

1. An explicit forward Euler integration scheme which gives rise to a constraint on the length of the time step to ensure stability. $\alpha_{n}$ is obtained as

$$
\alpha_{n}=\delta t \times K(T) \times G\left(\alpha_{n-1}\right)^{+\alpha_{n-1}}
$$

$\delta t$ being the time step.

2. An implicit backward Euler formulation, which is stable but requires a non-linear resolution at each time step. $\alpha_{n}$ is obtained by zeroing the residual

$$
R\left(\alpha_{n}\right)=\alpha_{n}-\alpha_{n-1}-\delta t \times K(T) \times G\left(\alpha_{n}\right)=0 .
$$

Equation (4) is usually zeroed with an iterative method, which may require the computation of the derivative of $d G / d \alpha$.

Difficulty.

During iterations, the Nakamura function $G$ and its derivative could be evaluated for various values of $\alpha$. Even non-physical values $(\alpha \notin[0,1])$ could be tested. In these cases the residual evaluation should return a value that allows further convergence.

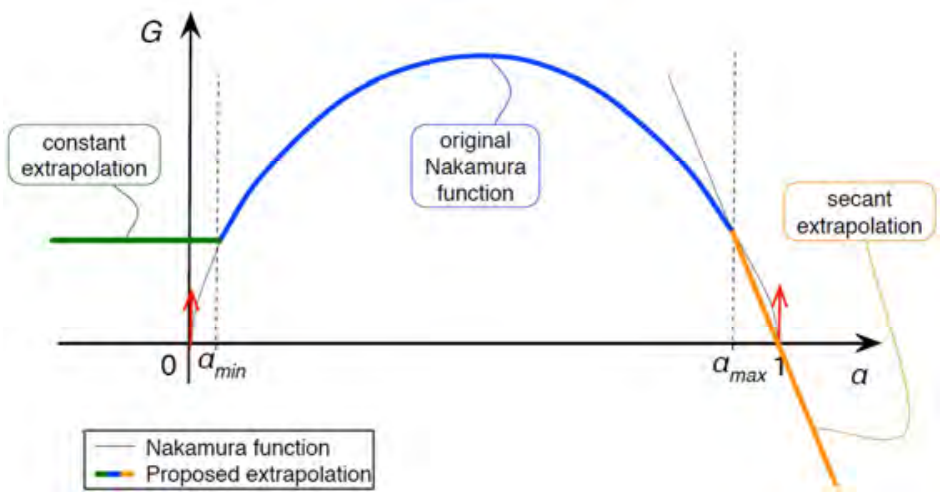

Figure 1. The original Nakamura function $G$ (equation (2)) is singular when $\alpha=0$ and when $\alpha=1$ (vertical tangent, represented by the red arrows). In the proposed modified extrapolation, a thresholding is performed below $\alpha_{\text {min }}$, and a linear extrapolation above $\alpha_{m}$ 


\subsection{Modified Nakamura Function}

The Nakamura function $G$ is modified in order to ensure robust numerical evaluation. As depicted in Figure 1, the original Nakamura function $G$ exhibits two singular points with infinite derivatives for $\alpha=0$ and $\alpha=1$.

These infinite derivatives may prevent convergence of the nonlinear solving of equation 4, especially with gradient methods. Two extrapolations are proposed on the function $G$.

- For values of $\alpha$ below a minimum threshold $\alpha_{\text {min }}$, $G(\alpha)$ is truncated to $\mathrm{G}\left(\alpha_{\text {min }}\right)$. This ensures:

(i) an artificial germination for the start of the initial crystallization,

(ii) real residual values (eq. 4) for $\alpha<0$ and

(iii) finite derivatives of $G(d G / d \alpha=0)$ in the vicinity of $\alpha=0$.

- For values of $\alpha$ above a maximum threshold $\alpha_{\max }$, $G(\alpha)$ is linearized such that it is continuous at $\alpha_{\max }$ and $G(1)=0$. This ensures:

(i) a smooth exponential decay of $\alpha$ towards $\alpha=1$, at the end of crystallization,

(ii) real residual values for $\alpha>1$ and

(iii) finite derivatives of $G$ in the vicinity of $\alpha=1$

\section{Results and Discussion}

In the following, in order to focus on the efficiency and robustness of numerical integration schemes, the study is performed with a constant

$$
K(T)=1 .
$$

The reader should keep in mind that in real industrial cases, the temperature field (and therefore $K(T)$ ) depends on space and time. Thus, equation (2) should be integrated in a global multiphysical integration scheme. For such applications, the reader may refer to references $[16,17,19,20,21,22]$.

Considering this degenerated case, with $K(T)=1$, the Nakamura equation (2) has an explicit analytical solution given by equation (1). This is helpful to study the convergence of the numerical schemes: in particular, the crystallization half-time for which $\alpha$ reaches 0.5 is known:

$$
t_{\frac{1}{2}}=(\ln 2)^{\frac{1}{n}}
$$

\subsection{Lower Truncation $\alpha_{\min }$}

The effect of the lower truncation $\alpha_{\text {min }}$, which accounts for the artificial germination, is first investigated. Two classic values of the Avrami index $n=2$ and $n=3$ are considered. A fine time step $\left(d t=\left[2.10^{-4}\right] s\right)$ is used to ensure converged time integration (see section 3.3). Figure 2 shows that as the value of $\alpha_{\text {min }}$ decreases, the crystallization half-time $t \frac{1}{2}$ converges towards the exact analytical solution.

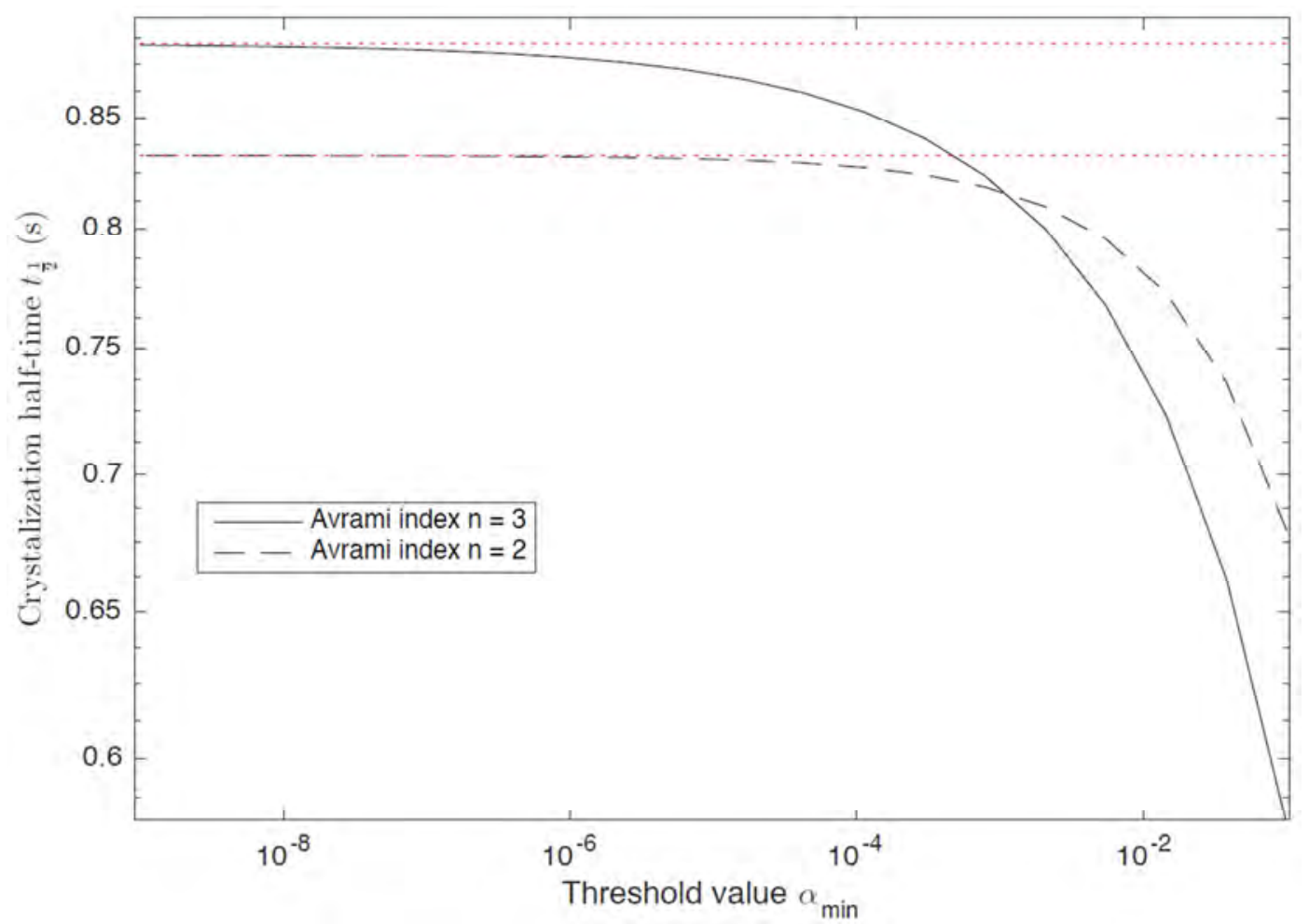

Figure 2. Convergence of the crystallization half-time $t_{\frac{1}{2}}$ versus the threshold value $\alpha_{\min }$. The horizontal dashed line represents the analytical values. $\alpha_{\text {min }}$ should be smaller than $10^{-6}$ in order to limit artificial germination and avoid inaccuracy and earlier predictions of crystallization effects. 
If $\alpha_{\min }$ is too high, the artificial germination is exaggerated and the crystallization time is underestimated. This parametric study suggests that the threshold value $\alpha_{\min }$ should be below $10^{-6}$ for Avrami index $n=2$, and below $10^{-8}$ for Avrami index $n=3$.

\subsection{Upper Truncation $\alpha_{\max }$}

As discussed in section 2.2, the time integration scheme may result in evaluating the Nakamura function $G$ for values of $\alpha$ above 1. This may occur in the cases of explicit formulations with large time steps or implicit formulations during the non-linear resolution (4). The upper linearization of $G$ ensures robustness in these cases.

With an explicit time integration with time steps $d t=0.2 \mathrm{~s}$, Figure 3 shows that a slight overshoot occurs.

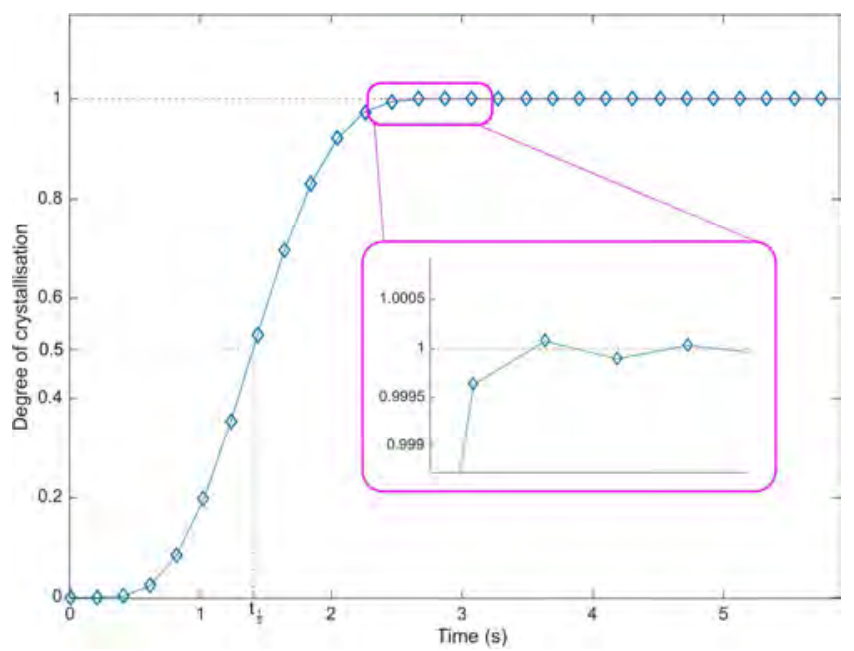

Figure 3. Time integration of the Nakamura kinetics using large time steps $(d t=0.2 s)$ and the explicit Euler scheme. The close-up shows that a slight overshoot is predicted. Nevertheless, the proposed modification of the Nakamura function ensures a robust later integration, with an exponential decay of $\alpha$ towards 1 .

Nevertheless, the extrapolation of the Nakamura function above $\alpha_{\max }$ forces $\alpha$ to approach 1 in further increments. Thus, the proposed extrapolation enables a robust time integration.

\subsection{Formulation Convergence}

Figure 4 shows the convergence of the crystallization half-time $t \frac{1}{2}$ versus the size of the time step $\delta t$ considered. It is advisable to use time steps no larger than $\left[5.10^{-3}\right] s$ to ensure accurate kinetics prediction ${ }^{1}$. In an industrial situation, faster time integration schemes (such as Crank Nicholson or Runge-Kutta) might also be used. Figure 5 is the convergence plot representing the relative error for both schemes versus inverse of time step. The classical first order convergence is

1 In the general case in which the Nakamura function $K(T)$ differs from unity, this time step recommendation should be divided by the function value. obtained with explicit or implicit scheme. Classical results in terms of stability can also be recovered. For instance, the resolution becomes instable for large time steps $(\mathrm{dt}>0.3 \mathrm{~s})$ with the explicit scheme.

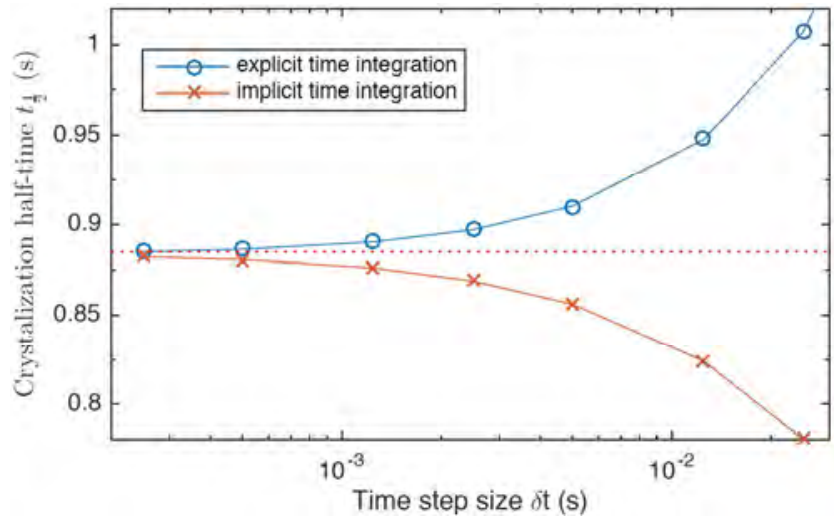

Figure 4. Convergence of the crystallization half-time $t \frac{1}{2}$ versus time step size $\delta t$. The horizontal dashed line represents the analytical value.

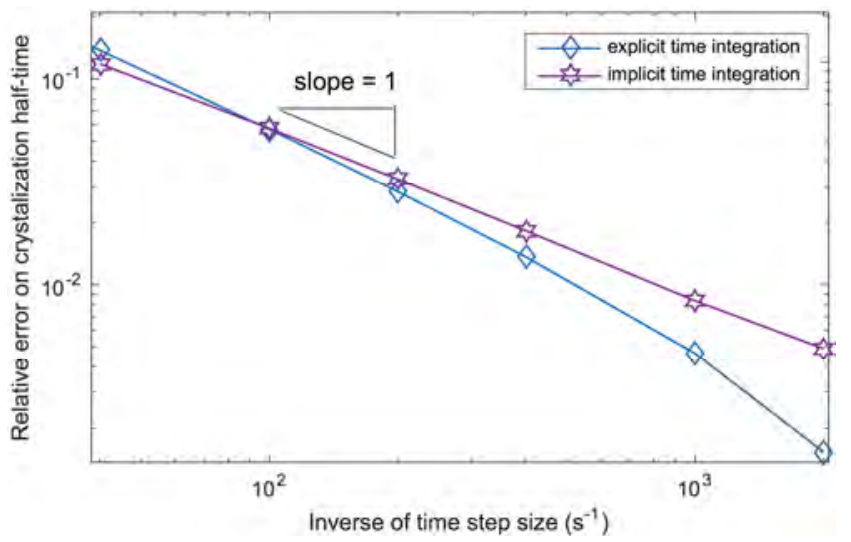

Figure 5. Convergence plot. Relative error between numerical and analytical crystalization half-time versus inverse of time step. The classical first order convergence for both scheme is recovered.

\section{Conclusion}

The crystallization of polymer macro-molecules is usually modelled by defining a macroscopic degree of crystallization $\alpha$, which varies between 0 and 1 . Classic modeling of crystallization kinetics is based on the Nakamura equation. This paper investigated the accuracy and robustness of the numerical time integration of Nakamura crystallization kinetics. The main contributions of this short communication are the following:

- For robust integration schemes, the Nakamura function should be truncated (i) for small and negative values of $\alpha$ and (ii) for values of $\alpha$ in the vicinity and above 1

- Guidelines concerning these truncation values are given. They are fairly restrictive $\left(\alpha_{\min }<10^{-6}\right)$.

- Guidelines for time stepping, in the case of standard forward and backward Euler schemes, are also provided. 


\section{References}

[1] ANSYS Inc. Polyflow technical specifications. Technical Report 2007.

[2] Autodesk. Moldflow Users manual 2016.

[3] Moldex 3D. Users Manual 2016.

[4] Baran I, Cinar K, Ersoy N, Akkerman R, Hattel JH. A Review on the Mechanical Modeling of Composite Manufacturing Processes. Archives of Computational Methods in Engineering 2016;

[5] Patel RM, Spruiell JE. Crystallization Kinetics During Polymer Processing -Analysis of Available Approaches for Process Modeling. Polymer Engineering and Science 1991; 31 (10): 730-738.

[6] Faraj J, Pignon B, Bailleul JL, Boyard N, Delaunay D, Orange G. Heat Transfer and Crystallization Modeling during Compression Molding of Thermoplastic Composite Parts. Key Engineering Materials 2015; 651-653: 1507-1512.

[7] Cogswell FN. Thermoplastic aromatic polymer composites. Woodhead: Cambridge UK., 1992.

[8] Boyard N. Heat Transfer in Polymer Composite Materials: Forming Processes. John Wiley \& Sons: Hoboken NJ, 2016.

[9] Avrami M. Kinetics of phase change. I General theory. The Journal of Chemical Physics 1939; 7: 1103.

[10] Haudin JM, Monasse B. Physico-chimie des polymères. Initiation à la chimie et à la physico-chimie macromoléculaires. chap. VII, Groupe Fran, 1996.

[11] Nakamura K, Katayama K, Amano T. Some aspects of nonisothermal crystallization of polymers. II. Consideration of the isokinetic condition. Journal of Applied Polymer Science 1973; 17 (4): 1031-1041.

[12] Billon N, Barq P, Haudin JM. Modelling of the Cooling of Semi-crystalline Polymers during their Processing. International Polymer Processing 1991; 6 (4): 348-355.

[13] Patel RM. Crystallization Kinetics Modeling of High Density and Linear Low Density Polyethylene Resins. Journal of Applied Polymer Science 2012; 124: 1542-1552.

[14] Tardif X, Pignon B, Boyard N, Schmelzer JW, Sobotka V, Delaunay D, Schick C. Experimental study of crystallization of PolyEtherEtherKetone (PEEK) over a large temperature range using a nano-calorimeter. Polymer Testing 2014; 36: 10-19.

[15] Neugebauer F, Ploshikhin V, Ambrosy J, Witt G. Isothermal and non-isothermal crystallization kinetics of polyamide 12 used in laser sintering. Journal of Thermal Analysis and Calorimetry 2016; 124 (2): 1-9.

[16] Levy A, Le Corre S, Sobotka V. Heat transfer and crystallization kinetics in thermoplastic composite processing. A coupled modelling framework. ESAFORM 19, Nantes, France, 2016.

[17] Pignon B, Tardif X, Lefèvre N, Sobotka V, Boyard N, Delaunay D. A new PvT device for high performance thermoplastics: Heat transfer analysis and crystallization kinetics identification. Polymer Testing 2015; 45: 152-160.

[18] Somé SC, Delaunay D, Faraj J, Bailleul JL, Boyard N, Quilliet $\mathrm{S}$. Modeling of the thermal contact resistance time evolution at polymer-mold interface during injection molding: Effect of polymers' solidification. Applied Thermal Engineering 2015; 84: $150-157$.

[19] Amado A, Wegener K, Schmid M, Levy G. Characterization and modeling of non-isothermal crystallization of Polyamide 12 and co-Polypropylene during the SLS process. 5th International Polymers and Moulds Innovations Conference, Ghent, Belgium, 2012; 207-216.

[20] Joshi SC, Lam YC. Integrated approach for modelling cure and crystallization kinetics of different polymers in 3D pultrusion simulation. Journal of Materials Processing Technology 2006; 174 (1-3): 178-182.

[21] Le Goff R, Poutot G, Delaunay D, Fulchiron R, Koscher E. Study and modeling of heat transfer during the solidification of semi-crystalline polymers. International Journal of Heat and Mass Transfer 2005; 48 (25-26): 5417-5430.

[22] Chan T, Isayev A. Quiescent polymer crystallization: Modeling and measurements. Polymer Engineering and Science 1994; 34 (6): 461-471.

[23] Kenny JM, Maffezzoli A, Nicolais R. A new kinetic model for polymer crystallization by calorimetric analysis. Thermochimica Acta 1993; 227 (December 1992): 83-95.

[24] Bessard E, De Almeida O, Bernhart G. Unified isothermal and non-isothermal modelling of neat PEEK crystallization. Journal of Thermal Analysis and Calorimetry 2014; 115 (2): 1669-1678.

[25] Velisaris CN, Seferis JC. Crystallization Kinetics of Polyetheretherketone (PEEK). Polymer Engineering and Science 1986; 26 (22): 1574. 